\section{ANÁLISIS DE LAS CARACTERÍSTICAS ORGANIZATIVAS DE LOS PROGRAMAS DE APOYO A ESTUDIANTES-DEPORTISTAS DE ÉLITE EN EL SISTEMA UNIVERSITARIO DE CATALUÑA}

\author{
ANÁLISE DAS CARACTERÍSTICAS ORGANIZACIONAIS DOS PROGRAMAS DE \\ APOIO A ESTUDANTES-ATLETAS DE ELITE NO SISTEMA UNIVERSITÁRIO DA \\ CATALUNHA
}

\section{ANALYSIS OF THE ELITE STUDENT-ATHLETES SUPPORT PROGRAMS' ORGANIZATIONAL CHARACTERISTICS IN CATALONIA'S HIGHER EDUCATION SYSTEM}

\section{Pau Mateu*, Anna Vilanova*, Eduard Inglés*}

\begin{abstract}
Palabras clave: Programas. Educación Superior. Organización y Administración.

Resumen: Este trabajo pretende analizar las características organizativas de los programas universitarios de apoyo a la carrera dual en Cataluña. La muestra se compuso de las diez universidades que disponían de programas de apoyo a la carrera dual. La recogida de datos se llevó a cabo mediante entrevistas a 13 coordinadores, así como a través del análisis del contenido existente en los sitios web de cada universidad. Los resultados exponen diferentes perfiles de estudiantes-deportistas presentes en los programas. Se han manifestado carencias en elementos como la disponibilidad de recursos, la planificación o la legislación vigente. Asimismo, factores externos como el profesorado o el sistema europeo de grados universitarios han mostrado tener una notable influencia. Se concluye que la voluntad política, un contexto económico favorable y la colaboración privada son elementos necesarios para avanzar en el desarrollo de los programas.
\end{abstract}

Palavras chave Programas. Educação superior. Organização e Administração.
Resumo: Este artigo tem como objetivo analisar as características organizacionais dos programas universitários de apoio à carreira dupla na Catalunha. A amostra foi composta pelas dez universidades que possuíam programas de apoio à carreira dupla. A coleta de dados foi realizada por meio de entrevistas com 13 coordenadores, bem como pela análise do conteúdo existente nos sites web de cada universidade. Os resultados mostram diferentes perfis de alunos-atletas presentes nos programas. Houve deficiências em elementos como a disponibilidade de recursos, planejamento ou legislação vigente. Além disso, fatores externos, como o corpo docente ou o sistema europeu de diplomas universitários, mostraram ter uma influência significativa. Conclui-se que a vontade política, um contexto econômico favorável e a colaboração privada são elementos necessários para avançar no desenvolvimento dos programas.

Abstract: This paper aims to analyze the organizational characteristics of higher education

Keywords:

Programs.

Education, Higher. Organization and Administration. programs supporting dual career in Catalonia. The sample included ten universities that had dual career support programs. Data collection was carried out through interviews with 13 coordinators as well as by analyzing the existing content of each university's websites. The results show different profiles of student-athletes linked to the programs. There have been deficiencies in elements such as the availability of resources, planning or current legislation. Moreover, external factors like teaching staff or the European system of university degrees have shown to have a significant influence. Findings point out that political will, a favorable economic context, and private contribution are necessary in order to advance in the programs' development.
* Institut Nacional d’Educació Física de Catalunya (INEFC). Barcelona, España. E-mail: pmateu@gencat.cat; anna.vilanova@inefcgiseafe.com; eduard.ingles@gencat.cat

Recebido em: 24/04/2018 Aprovado em: 06/11/2018

DOI: https://doi.org/10.22456/1982-8918.82235 (c) (1) () Licence 


\section{DIFICULTADES PARA COMPAGINAR DEPORTE DE ÉLITE Y ESTUDIOS}

Actualmente, el deporte ha adquirido tal nivel de profesionalización que, para llegar a la élite, los deportistas deben mantener una disciplina de entrenamiento que se alarga durante años, y que implica dedicar la mayor parte del día a la práctica deportiva desde la adolescencia, e incluso desde la infancia en determinadas disciplinas (VILANOVA; PUIG, 2013). Estas personas invierten en sus carreras deportivas durante edades decisivas en cuanto a su desarrollo vital y profesional (STAMBULOVA; WYLLEMAN, 2014), dedicando entre cinco y diez años para alcanzar el máximo nivel competitivo (ALFERMANN; STAMBULOVA, 2007).

Durante el tramo de edad entre los 20 y los 30 años, coincidiendo con la etapa de formación universitaria, la mayoría de deportistas se encuentran en su fase de máximo rendimiento (LÓPEZ DE SUBIJANA; CONDE; BARRIOPEDRO; GALLARDO, 2014). En estos años, los estudiantes-deportistas de élite ${ }^{1}$ a menudo se encuentran en un conflicto de intereses; por un lado, sus objetivos deportivos les someten a una carga de entrenamiento que requiere de una dedicación prácticamente equivalente a la de una profesión y, por otro lado, se enfrentan al reto de desenvolverse en un sistema educativo habitualmente estandarizado y restrictivo (EUROPEAN UNION, 2012). Si la conciliación no es posible, el estudiante-deportista de élite debe escoger entre continuar su carrera deportiva, o bien progresar en la educativa, renunciando a una de ellas (ÁLVAREZ, PÉREZ; GONZÁLEZ; LÓPEZ, 2014; GUIDOTTI, CORTIS; CAPRANICA, 2015).

Vilanova y Puig (2013) destacan que, para conseguir la inserción laboral una vez finalizada la etapa competitiva, el desarrollo de estrategias, como continuar estudiando durante la carrera deportiva, es una cuestión fundamental. De hecho, Pallarés, Azócar, Torregrosa, Selva y Ramis (2011) muestran que aquellos deportistas que escogen una trayectoria paralela -misma prioridad para el deporte y la formación- o convergente -el deporte es prioritario sobre la formación- perciben su transición fuera del deporte más satisfactoriamente que quienes siguen una trayectoria lineal -deporte como dedicación exclusiva.

Diversos estudios han determinado que la carrera dual (combinar la actividad deportiva con estudios) produce beneficios como evitar el abandono del deporte o de los estudios (BARON-THIENE; ALFERMANN, 2015), preparar a los estudiantes-deportistas para su etapa post-deportiva, previniendo posibles crisis de identidad y organizando su transición hacia el mercado laboral (VILANOVA; PUIG, 2014), o promover el bienestar individual mediante un estilo de vida equilibrado (AQUILINA, 2013; STAMBULOVA, ENGSTRÖM, FRANCK, LINNÉR; LINDAHL, 2015).

\subsection{Respuesta institucional: programas universitarios de apoyo a deportistas de élite}

El desarrollo de la carrera dual de los estudiantes-deportistas de élite ha supuesto una creciente preocupación para instituciones internacionales, estatales y regionales (EUROPEAN COUNCIL, 2013). Dichas instituciones, que no son ajenas a la trascendencia del deporte de élite (TÁBOAS-PAIS; CANALES-LACRUZ; REY-CAO; PERICH-SANCHO, 2015; VIANNA;

1 En este estudio, entendemos el término "deportista de élite" como un concepto neutro de deportista que entrena y compite a un elevado nivel. De esta forma, los términos "deportista de alto nivel" y "deportista de alto rendimiento" se referirán exclusivamente a aquellos contemplados en el Real Decreto 971/2007, mientras que la nomenclatura "deportista de alto nivel catalán" queda reservada para quienes cumplen con los supuestos establecidos en el Decreto 337/2002. 
LOVISOLO, 2009), han tratado de dar respuesta al problema orientando sus políticas hacia la integración de los estudiantes-deportistas de élite en el sistema educativo. En el año 2012, la Unión Europea publicó una guía con recomendaciones para que los Estados miembros, las federaciones deportivas y las instituciones educativas facilitaran la carrera dual de sus estudiantes-deportistas de élite (EUROPEAN UNION, 2012).

En España, la principal referencia legal en cuanto a la carrera dual figura en el Real Decreto 971/2007. En él se recogen medidas para promover la educación de los deportistas de élite, así como para facilitar su acceso a las diferentes ofertas formativas. Además, ciertas Comunidades Autónomas también han desarrollado leyes complementarias para favorecer la compaginación de estudios y deporte de élite. Organizaciones estatales como el Consejo Superior de Deportes y el Comité Olímpico Español han impulsado iniciativas para la formación y la orientación académico-laboral, entre otros (PROGRAMA DE ATENCIÓN AL DEPORTISTA DE ALTO NIVEL, 2017; OFICINA DE ATENCIÓN AL DEPORTISTA, 2017). Por otro lado, la Secretaria General de l'Esport de Cataluña ofrece el Servicio de Atención al Deportista (SAE), que facilita asesoramiento educativo y laboral, así como acompañamiento en la retirada deportiva (REGÜELA, 2011). El primer precedente estatal en cuanto a iniciativas universitarias de apoyo a la carrera dual lo encontramos en el año 1996, cuando la Universitat Autònoma de Barcelona desarrolló el primer programa universitario para ayudar, fundamentalmente a través de tutorías personalizadas, a combinar el deporte de élite con la educación superior (TORREGROSA; GONZÁLEZ, 2013).

En base a las evidencias mostradas sobre los beneficios que se derivan de la carrera dual, en los últimos años se han venido impulsando, desde el sistema universitario, programas de apoyo al colectivo de estudiantes-deportistas de élite (AQUILINA; HENRY, 2010; ISIDORI, 2016). Un trabajo de López de Subijana, Mascaró y Conde (2014) sobre 49 universidades españolas indica que, actualmente, hasta un $94 \%$ de éstas disponen de programas y servicios de apoyo a la carrera dual. Aun así, un informe de la Comisión Europea señala que, si bien se están desarrollando varias iniciativas en universidades europeas, existen carencias a nivel organizativo, y se muestra crítico con la falta de flexibilidad institucional para con los estudiantes-deportistas de élite (EUROPEAN COMMISSION, 2016).

Ante esta situación, Wylleman, Alfermann y Lavallee (2004) destacan la necesidad de conocer el funcionamiento de los programas de apoyo a estudiantes-deportistas de élite. Sin embargo, la literatura existente en relación a programas universitarios es escasa. En España, destaca la aportación de Mateos, Torregrosa y Cruz (2010), en la que se analiza la satisfacción de estudiantes-deportistas usuarios del programa Tutoresport-UAB. Por otro lado, el trabajo de Álvarez y López (2012) se centra en evaluar las necesidades los estudiantesdeportistas asociados al programa TUDAN de la Universidad de La Laguna para compaginar la actividad académica y deportiva. Sin embargo, no se han hallado referencias en cuanto a las características organizativas de los programas.

Así, el presente estudio pretende conocer las características organizativas de los programas universitarios de apoyo al colectivo de deportistas de élite en el sistema universitario de Cataluña. Se busca contribuir a la reflexión y, si cabe, a la implementación de mejoras en los programas por parte de las autoridades políticas y universitarias con respecto a los programas de apoyo a la carrera dual en la educación superior. 


\subsection{Análisis de características organizativas en programas}

La Teoría General de Sistemas de Von Bertalanffy (1968) define un sistema como un complejo de elementos en interacción, y fue pionera en la definición de un sistema abierto, caracterizado como un conjunto de intercambios de energía, materia, personas e información con el entorno. Sobre esta base, la Teoría de Sistemas Abiertos propuesta por Katz y Kahn (1978) aplica este concepto a las organizaciones, entendiéndolas como un sistema que se compone de tres componentes básicos: inputs/recursos, throughputs/procesos y outputs/productos; estos elementos generan un ciclo constante de feedback/retroalimentación. Esta teoría ha sido utilizada y adaptada al contexto deportivo para explicar fenómenos tales como los factores de la política deportiva que conducen al éxito deportivo internacional (DE BOSSCHER; DE KNOP; VAN BOTTENBURG; SHIBLI, 2006). En esta línea, las principales dimensiones que conforman el ciclo de cualquier programa siguen una lógica determinada por una serie de elementos clave: población objetivo, recursos, procesos, productos/servicios, impactos/feedback y factores contextuales (MCLAUGHLIN; JORDAN, 2015; BLASCO, 2009). En consecuencia, estos son los elementos que se han considerado para conocer las características organizativas de los programas universitarios de apoyo a la carrera dual en Cataluña.

\section{MÉTODO}

Siguiendo un muestreo intencional por criterio (SPARKES; SMITH, 2014), la muestra se compuso de las diez universidades catalanas que disponían de programas de apoyo a la carrera dual. Ocho de ellas eran de carácter público, y dos de titularidad privada. Hasta siete universidades se ubicaban en la provincia de Barcelona, mientras que las otras tres se encontraban en las capitales de las provincias de Tarragona, Lleida y Girona. Un total de 13 personas encargadas de coordinar los programas participaron en el estudio, de las cuales ocho eran hombres y cinco mujeres. Con el fin de identificar a las universidades en la redacción de los resultados, así como los fragmentos textuales de las entrevistas realizadas, se asignó un código a cada uno de los coordinadores. Así, la persona que coordina el programa de la Universidad 1 recibió el código C1, a la encargada del programa de la Universidad 2 se le asignó el C2, y así sucesivamente.

Se combinaron dos técnicas de recolección de datos. Por un lado, se elaboró una guía de entrevista (QUIVY; CAMPENHOUDT, 2005), compuesta por preguntas relativas a poblaciones objetivo, recursos, actividades, productos, impactos y factores contextuales de los programas. Por otro lado, se llevó a cabo un análisis del contenido disponible sobre los programas en los sitios web de las universidades seleccionadas. Este enfoque es conveniente para estudiar textos con contenido programático (HEINEMANN, 2008).

Todas las entrevistas fueron llevadas a cabo por el primer autor. Los participantes fueron informados sobre los objetivos del estudio y el tratamiento ético de los datos. Once de ellos firmaron un documento de consentimiento informado, los dos restantes dieron su consentimiento de manera verbal. Las entrevistas tuvieron una duración media de 45 minutos, y fueron grabadas para asegurar su validez. En cuanto al análisis del contenido web, la unidad de análisis empleada fue la página web de cada universidad. Los datos se analizaron a través del software QSR NVivo 11, mediante el cual se identificaron fragmentos significativos en relación a los elementos valorados en este estudio. 


\section{RESULTADOS}

\subsection{Población objetivo}

Los estudiantes-deportistas de élite, al igual que el resto de alumnado regular, pueden escoger hasta 430 carreras diferentes en el sistema universitario catalán. Los estudios vinculados a las Ciencias del Deporte, así como los vinculados a las ciencias sociales y jurídicas, se encuentran entre los más escogidos por el colectivo de estudiantes-deportistas de élite (C9).

Los programas estudiados contemplan diferentes tipologías de deportistas de élite -estatales, regionales, universitarios y otros- como público objetivo. La tabla 1 muestra los perfiles establecidos como criterios de inclusión para los programas.

Tabla 1 - Población objetivo de los programas

\begin{tabular}{llc}
\hline & Tipología desportistas élite & Programas \\
\hline \multirow{3}{*}{ Estatal } & Alto Nivel / Alto Rendimiento (RD 971/2007) & 9 \\
& Planes ADO / ADOP & 8 \\
& Selecciones nacionales & 7 \\
\hline Regional & Alto Nivel Catalán (D 337/2002) & 8 \\
\hline \multirow{2}{*}{ Universitario } & Participantes Mundiales Universitarios / Universiadas & 5 \\
& Medalistas Campeonatos de España Universitarios & 8 \\
\hline Otras & & 5 \\
\hline
\end{tabular}

Fuente: elaboración propia.

Inicialmente, únicamente se contemplaban los deportistas de élite que pudiesen certificar su condición de Alto Nivel o Alto Rendimiento, su pertenencia a los planes ADO/ADOP o bien convocatorias a selecciones nacionales. Los deportistas de carácter universitario fueron los últimos en ser incorporados como criterios de admisión de los programas, donde tienen una importante presencia: "es el grueso, vendría a ser el $80 \%$ de la gente de nuestro programa" (C9.2). Existen otras tipologías que progresivamente se están incorporando a los programas: deportistas profesionales y semi-profesionales; convocados por selecciones autonómicas, etcétera. "hay de ligas, de 3를 división, que también los atendemos, porque no están registrados oficialmente, pero tienen una carga de entrenamientos y competiciones alta" (C8.2).

El criterio para permitir el acceso a estos otros tipos de deportistas a los programas es variable. La Universidad 9 requiere haber establecido previamente acuerdos con organizaciones como, por ejemplo, clubes deportivos o federaciones. Otras universidades, sin embargo, se muestran flexibles a la hora de incorporar en sus programas a estudiantes-deportistas que no cumplen con los criterios de inclusión predefinidos: "muchos no reúnen los requisitos, pero no queremos ser un impedimento para que el estudiante practique deporte, aunque no sea al máximo nivel" (C7).

\subsection{Recursos económicos y humanos}

El presupuesto de todos los programas se incorpora en el de los departamentos universitarios que los desarrollan: "lo tenemos todo integrado, no hay partida específica" (C8.1), que generalmente son los Servicios de Deportes. Años atrás, órganos universitarios y entidades 
externas destinaban partidas específicas: "por parte del Consejo Superior de Deportes había una aportación económica [...]. El Consejo Social de la Universidad 5 doblaba la misma aportación que daba el Consejo" (C5.1). Sin embargo, recortes presupuestarios terminaron por suprimir estas partidas: "con el tema de la crisis se dejó de subvencionar el programa" (C1). Así, actualmente los programas se financian por medio los departamentos en los que se gestionan y, en menor medida, del Centro de Alto Rendimiento ${ }^{2}$ (CAR). Esta entidad otorga una subvención variable en función de la cantidad de estudiantes-deportistas inscritos a los programas que estén reconocidos como de Alto Nivel Catalán. No obstante, la crisis económica también ha afectado al importe otorgado: "EI CAR da una cantidad de dinero que antes era más elevada y después, con los recortes, se ha reducido prácticamente a la mitad” (C4).

Los salarios del personal encargado de la gestión de los programas, así como las compensaciones económicas a los tutores y tutoras, son los gastos más habituales: "Al tutor se le hace un pequeño pago. Estamos hablando de unos $100 €$ o así, antiguamente, cuando había más ayudas, podíamos dar más" (C3). El programa de la Universidad 1, por su parte, invierte recursos en otros conceptos:

Una parte importante del dinero se utiliza para comprar material, que se da a todos los deportistas. La otra parte se da a los deportistas que representan a la Universidad 1 en campeonatos [...]. En función de las medallas, se da una cantidad más alta, o no. (C1).

En cuanto a recursos humanos, el personal trabaja en los programas se divide en, por un lado, las personas que coordinan el programa y, por el otro, las que ejercen tareas de tutorización sobre los y las estudiantes-deportistas.

Las funciones de las personas que coordinan los programas son, fundamentalmente, de carácter administrativo: "Básicamente son burocráticas. Viene el estudiante, llenamos la solicitud, revisamos que sea deportista de élite, y después lo derivamos a la facultad" (C7). En las universidades que ofrecen uso gratuito de instalaciones deportivas a sus estudiantesdeportistas, pero que no disponen de un espacio propio, las personas que coordinan los programas establecen acuerdos con entidades tales como ayuntamientos o clubes deportivos (C1, C3, C7). Asimismo, también informan al profesorado sobre la existencia y condiciones de los programas: "si hay algún profesor que tiene dudas, se pone en contacto conmigo" (C9.1). Además, ejercen tareas de mediación: "Si un profesor dice «no, yo a este (deportista) no le cambio el examen porque no me viene bien», yo le digo «ya, pero dentro del tiempo que tengas, se lo has de cambiar»" (C3). Por último, se encargan de hacer un seguimiento de la trayectoria académica y deportiva de sus personas inscritas a los programas; en general, el seguimiento se limita a deportistas de Alto Nivel Catalán, dado que el Centro de Alto Rendimiento exige un informe anual como condición para otorgar sus subvenciones.

El colectivo de tutores está conformado por profesorado de las universidades. En ocasiones, ocupan cargos directivos para agilizar los trámites y ejercer una mayor influencia sobre el resto de docentes: "Algunas veces [...] muchos de los tutores y tutoras son los mismos jefes de estudios, lo que facilita después muchas cosas" (C4). Entre sus funciones figuran el acompañamiento, la orientación académica y la resolución de dudas a estudiantes-deportistas, así como la interlocución entre éstos y otros miembros del profesorado para resolver problemas o solicitar adaptaciones académicas: "el tutor es quien habla con compañeros suyos, profesores, 
para ayudar" (C2). En la Universidad 3, también facilitan apuntes de las clases a las que no han podido acudir los estudiantes-deportistas.

La mayor parte de las universidades disponen de una sola persona encargada de coordinar sus programas. En cuanto a los tutores y tutoras, su número varía significativamente entre universidades: mientras que la Universidad 7 cuenta con apenas cuatro tutores, la Universidad 4 dispone de aproximadamente 40. Esta información, así como la referente a la cantidad de estudiantes-deportistas inscritos a cada programa, se encuentra detallada en la tabla 2.

Tabla 2 - Personal asociado a los programas. $\left({ }^{*}=\right.$ valores aproximados $)$

\begin{tabular}{cccc}
\hline Universidad & Coordinación & Tutores/as & Estudiantes/desportistas \\
\hline Universidad 1 & 1 & $15-20^{*}$ & $35-40^{*}$ \\
Universidad 2 & 1 & $30^{*}$ & $40^{*}$ \\
Universidad 3 & 1 & $18-20^{*}$ & 22 \\
Universidad 4 & 1 & $40^{*}$ & $70-75^{*}$ \\
Universidad 5 & 2 & $10^{*}$ & 7 \\
Universidad 6 & 1 & $30^{*}$ & $120-130^{*}$ \\
Universidad 7 & 1 & 4 & 11 \\
Universidad 8 & 2 & No informa & $60-70^{*}$ \\
Universidad 9 & 2 & $10-15^{*}$ & 120 \\
Universidad 10 & 1 & 22 & 63 \\
\hline
\end{tabular}

Fuente: elaboración propia.

\subsection{Procesos: actividades, estructura y relaciones interorganizativas}

Las acciones realizarse desde los programas están, en mayor o en menor grado, definidas. La mayoría de programas $(\mathrm{C} 1, \mathrm{C} 3, \mathrm{C} 6, \mathrm{C} 8 \mathrm{C} 9, \mathrm{C10})$ calendarizan las acciones a realizar, o bien tienen sus procesos claramente delimitados. Al inicio del curso, se realizan campañas de difusión: "hacemos publicidad al inicio del curso, que todos los interesados en el tema de deportistas de élite lo puedan pedir" (C1). Al mismo tiempo, se inician las inscripciones a los programas, que suelen tener un plazo temporal definido, si bien algunas universidades (C7, C8, C9) se muestran flexibles en este sentido. A continuación, se asignan tutores a los estudiantesdeportistas, tarea reservada a posiciones jerárquicas relevantes: "contactamos con la dirección de cada facultad o con los jefes de estudios, y les decimos «mira, tenemos estos estudiantes en el programa, necesitamos un tutor de vuestro ámbito de estudio» Entonces responden, la facultad nos los asigna" (C4). Algunos programas (C1, C6, C10) establecen un protocolo más o menos estricto de encuentros entre tutor y estudiante-deportista, pero no es el caso habitual. En cambio, sí es habitual pedir a los estudiantes-deportistas que justifiquen los motivos por los que deben adaptárseles ciertas actividades académicas: "les decimos "pásanos los partidos que tendrás [...]", para ver si coinciden con el día de los exámenes" (C8.1). Cuando termina el curso, ciertos programas $(\mathrm{C} 6, \mathrm{C} 10)$ hacen una valoración final mediante cuestionarios de seguimiento académico y deportivo, si bien no es una acción que esté ampliamente extendida. Cabe señalar que algunos programas no planifican sus actuaciones, actúan conforme surge la necesidad: "Hasta donde yo sé, no hay nada fijado de antemano" (C2).

En cuanto a su estructura organizativa, la mayoría de programas $(\mathrm{C} 1, \mathrm{C} 2, \mathrm{C} 3, \mathrm{C4}, \mathrm{C5}$, C7, C9) están vinculados a los diferentes Servicios de Deportes de las universidades que, a 
su vez, dependen de estructuras como los vicerrectorados. Respecto a las comunicaciones, el canal más utilizado es el correo electrónico, seguido del teléfono. En las universidades más pequeñas, son habituales los encuentros en entornos informales: "Vía mail, teléfono... o en el bar. Somos un sitio muy pequeño. En la Universidad 7, todo el profesorado, todo el mundo nos conocemos" (C7).

Acerca de las relaciones interorganizativas, los programas establecen acuerdos formales e informales con diferentes entidades. Entre las públicas, todos los programas tienen convenios con el Centro de Alto Rendimiento, si bien algunos han tratado con otras: "pueden ir a las instalaciones del Ayuntamiento, o de clubes, porque la mayoría de instalaciones que usan los clubes también son del Ayuntamiento" (C1). En cuanto a entidades privadas, destacan los clubes deportivos y los gimnasios, a los que acuden los programas de universidades sin instalaciones deportivas propias, para que permitan el acceso a sus estudiantes-deportistas: "Tenemos un convenio con un club de atletismo, por ejemplo. Quien viene de fuera, puede entrenar en sus pistas" (C3). La Universidad 9, por su parte, ha establecido un convenio con un club de fútbol profesional para que sus deportistas se beneficien de los servicios del programa, a pesar de que sus criterios de inclusión sean diferentes a los preestablecidos: "tenemos un acuerdo con el Club33, a través de su plan de acción tutorial" (C9.2).

\subsection{Servicios académicos, deportivos y económicos}

Los programas ofrecen diferentes servicios académicos, deportivos y económicos. A nivel académico, la tutorización personalizada es el servicio más común, así como la adaptación de actividades académicas: "Ofrecemos tutorías, asesoría, cambio de exámenes y prácticas siempre que se pueda" (C9.1). Otro servicio académico ampliamente extendido es la obtención de créditos ECTS, con los que los estudiantes-deportistas pueden convalidar asignaturas optativas: "damos créditos libres, ahora se llaman ECTS. Les damos un crédito por curso, que son 25 horas" (C5.1). Ejemplos de otros servicios académicos son la cesión de materiales didácticos (C3), o la evaluación online (C8). A nivel deportivo, el uso gratuito de instalaciones deportivas -propias o ajenas- es el servicio más extendido: "tienen acceso gratuito a nuestras instalaciones deportivas. Gratuito para ellos, para nosotros es un coste indirecto, pero con la ayuda del CAR lo revertimos" (C4). En cuanto a otros servicios deportivos, cabe mencionar el apoyo psicológico $(\mathrm{C} 3, \mathrm{C} 6)$ y la cesión de material deportivo $(\mathrm{C} 1)$. Únicamente un programa ofrece ayudas económicas a sus estudiantes-deportistas, bajo la condición de que participen en competiciones universitarias: "si tú representas a la Universidad 1 pues, una parte económica ya te corresponde, ¿de acuerdo? Si no consigues resultados, tienes una parte económica más pequeña que si ganas medallas" (C1).

\subsection{Feedback: calidad percibida, impactos y evaluación}

Las principales fuentes para obtener retroalimentación acerca del desarrollo de los programas son, por un lado, la calidad percibida por las personas implicadas en los mismos y, por otro lado, los impactos generados por su aplicación. Las personas que coordinan los programas impulsados por la Universidad 6 y la Universidad 10 son los que disponen de una información más amplia sobre la calidad percibida de sus programas, tanto por parte de

3 Se ha modificado el nombre del club, con el objetivo de garantizar su anonimato. 
estudiantes-deportistas como de tutores y tutoras. Esto se debe a que son los únicos programas que han implementado un sistema de seguimiento mediante encuestas e informes regulares. El resto de programas, sin embargo, obtienen la información sobre la calidad percibida por otras vías: "hablando con estudiantes que están en el programa, dicen «sí, sí, con el programa me va muy bien. No me preocupo, es un dolor de cabeza menos". Y eso, al final, es lo que queremos, ¿no?” (C3). En general, se percibe que los estudiantes-deportistas están satisfechos y que los programas funcionan: "tenemos un servicio de atención de quejas y nunca hemos recibido ninguna de deportistas de élite protestando que, por su condición, les ha pasado algo" (C7).

Al valorar los impactos producidos por los programas, a nivel de resultados académicos y deportivos obtenidos por estudiantes-deportistas, el seguimiento es variable (C3, C4, C6, C10), si bien siempre se realiza en aquellos subvencionables por el CAR. En todo caso, más allá de valorar o no estos resultados, las personas que coordinan los programas creen que el principal impacto a corto plazo debe ser hacer efectiva la compaginación entre el ámbito educativo y el deportivo: "queremos que el deportista pueda realizar la práctica deportiva a su máximo nivel, y lo pueda compaginar con su carrera académica, aunque sea más lenta... pero que lo pueda hacer" (C2). A largo plazo, se destaca que los programas contribuyen a evitar el abandono de los estudios universitarios: "si no les ayudásemos, muchos de estos deportistas tendrían que dejar los estudios, o cambiar de carrera" (C4).

Los programas también varían en cuanto a su evaluación. Algunos se revisan con regularidad: "Vamos leyendo cada año el programa por si hay que modificar alguna cosa que lo mejore" (C1). En cambio, otros programas que no se evalúan, ya sea porque las personas que los coordinan no lo creen oportuno, o bien porque no se dispone de recursos que permitan realizar un análisis e/o implementar mejoras: "Alguna vez sí que hemos pensada «lo tendríamos que revisar...». Pero de momento, estamos como estamos" (C5.2). Entre otros aspectos a mejorar, los coordinadores señalan la ampliación de criterios para la inclusión de estudiantesdeportistas $(\mathrm{C} 1, \mathrm{C} 3, \mathrm{C} 8)$, la necesidad de mayores recursos económicos y humanos (C1, C2, C3, C4, C5, C9), de mejorar la coordinación interna y planificar las acciones (C2, C3, C5, $\mathrm{C} 8, \mathrm{C}$ ), la posibilidad de ofrecer más servicios académicos y económicos $(\mathrm{C} 2, \mathrm{C} 3, \mathrm{C} 5$,) el establecimiento de normativas a nivel universitario y/o legal (C1, C2, C5, C7, C8, C9, C10), o la misma evaluación de los programas (C2, C4, C7, C9).

\subsection{Factores contextuales}

Los programas se encuentran bajo la influencia de factores externos que determinan el contexto particular de cada uno de ellos. La dificultad a nivel económico es el más comentado, dado que provocó la limitación o supresión de aportaciones financieras a los programas por parte de organizaciones públicas. Otro elemento destacado es la sensibilidad del profesorado universitario hacia la carrera dual. Si bien la colaboración del profesorado con los programas se percibe como aceptable, en algunas universidades todavía existen reticencias: "somos una universidad tradicional, con profesorado de gran edad en varias facultades [...]. Con esta gente, hablar de deportistas de élite, cuesta" (C9.2). El marco legal al que se acogen los programas es también significativo para los coordinadores, que mayoritariamente perciben como escaso y poco específico, lo que conduce a interpretaciones subjetivas: "La Ley del Deporte, artículo tal... algo hay, pero es muy genérico, no hay nada concreto" (C3). En parte, las carencias a 
nivel legal se interpretan como falta de voluntad política por parte de las instituciones públicas, así como de las mismas universidades. La implantación de los nuevos planes de estudios en la universidad, derivada del desarrollo del EEES, también es un factor relevante. La estructura de los grados exige que los estudiantes acudan a un $80 \%$ de las clases de manera presencial, en contraste con las antiguas licenciaturas, que eran más flexibles en este sentido: "con el nuevo plan de estudios, los problemas se han multiplicado exponencialmente. El tema de la asistencia obligatoria es el problema número uno" (C9.1). Disponer de métodos de enseñanzaaprendizaje y de evaluación a distancia es, de acuerdo con las coordinadoras de la Universidad 8, un elemento a considerar: "con la docencia online las dificultades asociadas al espacio y el tiempo están superadas" (C8.2). Por último, desde algunos programas se ha hecho referencia al tamaño de las universidades en cuanto a la facilidad para tratar con miembros del profesorado, estudiantes-deportistas, tutores, etcétera: "hablamos incluso en el bar. Somos una universidad pequeña, y todos nos conocemos. En universidades grandes, si has de hablar con alguien, es complicado" (C7).

\section{DISCUSIÓN Y CONCLUSIONES}

Este trabajo se centra en conocer las características organizativas de los programas universitarios de apoyo a la carrera dual en Cataluña, tal y como sugerían Wylleman, Alfermann, y Lavallee (2004). De acuerdo con lo establecido por Aquilina y Henry (2010), la población objetivo de los programas engloba diferentes tipologías formales de deportistas de élite. Sin embargo, se ha evidenciado que los requisitos de acceso se están flexibilizando progresivamente, permitiendo la entrada a cada vez más estudiantes-deportistas que no siempre cumplen los estándares burocráticos habituales.

En cuanto a los recursos económicos de los programas, no existen presupuestos específicos dado que se integran en los departamentos que los gestionan. En consecuencia, no es posible determinar los costes directos de los programas; aspecto que autores como Blasco (2009) o McLaughlin y Jordan (2015) definen como necesario para evaluar cualquier programa. Sin embargo, sí se han detectado otros inputs señalados por los autores citados, como la cantidad de recursos humanos asociados a los programas, considerando personal de coordinación y tutores. La falta de planificación en ciertos programas confirma algunas de las críticas de la Comisión Europea (EUROPEAN COMMISSION, 2016), si bien se ha mostrado que, en varios programas, las actividades sí están estructuradas.

Se han identificado servicios de apoyo de tipo académico, deportivo y económico (AQUILINA; HENRY, 2010, LOPEZ DE SUBIJANA; MASCARÓ; CONDE, 2014). En el presente estudio, destacan aquellos de carácter académico que ofrecen flexibilidad en aspectos como fechas de realización de exámenes y entrega de trabajos, así como la tutorización personalizada de estudiantes-deportistas. Se trata de servicios que varias publicaciones e informes de carácter estatal (ÁLVAREZ; LOPEZ, 2012) e internacional (EUROPEAN UNION, 2012; ISIDORI, 2016) señalan como apropiados para el apoyo a la carrera dual.

La mayor parte de coordinadores perciben que los programas funcionan correctamente, a pesar de las dificultades en cuanto a la disponibilidad de recursos. Asimismo, exponen que los estudiantes-deportistas adscritos a sus programas están, en general, satisfechos, en coincidencia con investigaciones previas sobre programas desarrollados en Cataluña 
(MATEOS; TORREGROSA, 2010; MATEU; VILANOVA; ANDRÉS, 2017). Algunos programas evalúan su situación con cierta regularidad -al fin de cada curso, por lo general- en línea con las propuestas de Blasco (2009). A través de cuestionarios, entrevistas, u otros métodos menos ortodoxos, la mayoría de programas obtienen información regular, a través de la cual pueden implementar, si es posible, ciertas mejoras. En todo caso, y en coincidencia con BaronThiene y Alfermann (2015), la percepción general es que los programas de apoyo a la carrera dual son imprescindibles para facilitar la compaginación de deporte y estudios, lo que evita que los estudiantes-deportistas abandonen prematuramente sus carreras académicas.

Más allá de las dificultades en materia de recursos, ya mencionadas, se ha reportado, desde algunos programas, cierta falta de empatía y flexibilidad por parte de algunos miembros del profesorado, especialmente con los más veteranos. Este comportamiento, que no valora el contexto y las necesidades concretas del colectivo de estudiantes-deportistas, representa un obstáculo en el desarrollo tanto de la actividad deportiva, como de la académica que, tras la retirada de la competición, puede facilitar la inserción laboral (VILANOVA; PUIG, 2014). Esto coincide con hallazgos obtenidos en estudios españoles que analizan la percepción de estudiantes-deportistas (ÁLVAREZ; LÓPEZ, 2012; ÁLVAREZ et al., 2014). Se trata de una revelación importante, dado que la involucración del profesorado en un factor determinante en el desarrollo efectivo de programas de apoyo a la carrera dual (STAMBULOVA et al., 2015). Asimismo, las personas que coordinan los programas también han resaltado la importancia de mejorar el marco legal existente respecto a las carreras duales de los estudiantes-deportistas de élite, al considerarlo limitado y, en ocasiones, ambiguo. En este sentido, coinciden con recomendaciones establecidas en informes de ámbito europeo (EUROPEAN COMMISSION, 2016).

Este trabajo muestra el estado actual de las características organizativas de los programas universitarios de apoyo a la carrera dual que se llevan a cabo en Cataluña, aportando una visión interna que, al mismo tiempo, está necesariamente ligada al contexto. Los resultados se han obtenido a partir de la visión personal de los responsables de los distintos programas y, aunque se trata de una opinión protagonista y directa, futuros estudios evaluando la percepción de estudiantes-deportista de élite participantes o de tutores, podrían enriquecer la información obtenida. Por otro lado, que el presente trabajo se fundamenta en el hecho que las características de los programas están influenciadas por el entorno, abre las puertas a la posibilidad de analizar y comparar las características de programas desarrollados en otras regiones del Estado, así como en otros países de diferentes contextos culturales.

En definitiva, los resultados expuestos nos conducen a sugerir que, si se pretende avanzar en el desarrollo y mejora de dichos programas y, por ende, de las condiciones del colectivo de estudiantes-deportistas de élite, las instituciones públicas, así como los órganos de dirección de las mismas universidades, no pueden mantenerse al margen. Desarrollar el marco legal respecto al colectivo de estudiantes-deportistas de élite, así como dotar de recursos a los programas de apoyo a la carrera dual es una cuestión, en parte, de voluntad política. Ahora bien, no puede obviarse que el contexto de crisis económica ha condicionado sobremanera la capacidad de los organismos públicos para asignar una mayor cantidad de recursos. Por otro lado, cabe señalar la ausencia de financiación por parte de instituciones privadas y empresas que, sin duda, podrían aportar parte de los recursos necesarios para los programas universitarios de apoyo a la carrera dual, tal y como ya hacen en otras iniciativas vinculadas al deporte de élite como el programa ADO de becas a deportistas de nivel olímpico. Tal y como 
señala un coordinador de la Universidad 9: "el deporte y la actividad física, en ocasiones, no se consideran importantes. Necesitaríamos una mayor complicidad por parte de todas las organizaciones" (C9.2). Finalmente, las estrategias y políticas deben trazarse basándose en análisis empíricos para dar respuesta a las necesidades reales.

\section{REFERENCIAS}

ALFERMANN, Dorothee; STAMBULOVA, Natalia. Career transitions and career termination. In: TENENBAUM, Gershon; EKLUND, Robert. Handbook of Sport Psychology. New York: Wiley, 2007. p. $712-736$.

ÁLVAREZ, Pedro; LÓPEZ, David. Armonización entre proceso de aprendizaje y práctica deportiva en universitarios deportistas de alto nivel. Cultura, Ciencia y Deporte, v. 7, n. 21, p. 201-212, 2012.

ÁLVAREZ, Pedro; PÉREZ, David; GONZÁLEZ, Manuel; LÓPEZ, David. La formación universitaria de deportistas de alto nivel : análisis de una compleja relación entre estudios y deporte. Retos. Nuevas tendencias en Educación Física, Deporte y Recreación, v. 26, n. 2, p. 94-100, 2014.

AQUILINA, Dawn. A Study of the Relationship Between Elite Athletes' Educational Development and Sporting Performance. The International Journal of the History of Sport, v. 30, n. 4, p. 374-392, 2013.

AQUILINA, Dawn; HENRY, lan. Elite athletes and university education in Europe: a review of policy and practice in higher education in the European Union Member States. International Journal of Sport Policy and Politics, v. 2, n. 1, p. 25-47, 2010.

BARON-THIENE, Anna; ALFERMANN, Dorothee. Personal characteristics as predictors for dual career dropout versus continuation - A prospective study of adolescent athletes from German elite sport schools. Psychology of Sport and Exercise, v. 21, p. 42-49, 2015.

BLASCO, Jaume. Avaluació del disseny. Barcelona: Ivàlua, 2009.

DE BOSSCHER, Veerle; DE KNOP, Paul; VAN BOTTENBURG, Maarten; SHIBLI, Simon. A Conceptual Framework for Analysing Sports Policy Factors Leading to International Sporting Success. European Sport Management Quarterly, v. 6, n. 2, p. 185-215, 2006.

EUROPEAN COMMISSION. Study on the Minimum Quality Requirements for Dual Career Services. Luxembourg: Publications Office of the European Union, 2016.

EUROPEAN COUNCIL. Conclusions of the Council and of the Representatives of the Governments of the Member States, meeting within the Council, on dual careers for athletes. Brussels, 2013. Disponible en: <http://eur-lex.europa.eu/legal-content/ES/TXT/ PDF/?uri=OJ:C:2013:168:FULL\&from=ES> Acceso en: 04 may 2017.

EUROPEAN UNION. EU Guidelines on Dual Careers of Athletes. Recommended policy actions in support of dual careers in high-performance sport. Brussels, 2012. Disponible en: $<h t$ tp://ec.europa.eu/sport/library/documents/dual-career-guidelines-final_en.pdf> Acceso en: 04 may 2017. 
GUIDOTTI, Flavia; CORTIS, Cristina; CAPRANICA, Laura. Dual Career of European StudentAthletes: A Systematic Literature Review. Kinesiologia Slovenica, v. 21, n. 3, p. 5-20, 2015.

HEINEMANN, Klaus. Introducción a la metodología de la investigación empírica en las Ciencias del Deporte. Barcelona: Paidotribo, 2008.

ISIDORI, Emanuele. The dual career of student athletes and the quest for a personalized tutorship model. International Journal of Novel Research in Education and Learning, v. 3, n. 2, p. 9-15, 2016.

KATZ, Daniel; KAHN, Robert. The Social Psychology of Organizations. New York: Wiley, 1978.

LÓPEZ DE SUBIJANA, Cristina; CONDE, Elena; BARRIOPEDRO, María; GALLARDO, Leonor. Eficacia en la aplicación de las medidas de formación para los deportistas de alto nivel en el territorio español. Revista de Psicología del Deporte, v. 23, n. 1, p. 41-48, 2014.

LÓPEZ DE SUBIJANA, Cristina; MASCARÓ, F; CONDE, Elena. Los servicios de asesoramiento a los deportistas de élite en las universidades españolas. Kronos, v. 13, n. 2, 2014.

MATEOS, Marina; TORREGROSA, Miquel; CRUZ, Jaume. Evaluation of a career assistance programme for elite athletes: satisfaction levels and exploration of career decision making and athletic-identity. Kinesiologia Slovenica, v. 16, n. 1-2, p. 30-43, 2010.

MATEU, Pau; VILANOVA, Anna; ANDRÉS, Ana. Evaluación de un programa de carrera dual en el INEFC de Barcelona: niveles de satisfacción y propuestas de mejora. Revista Española de Educación Física y Deportes, v. 418, n. Supl, p. 121-128, 2017.

MCLAUGHLIN, John; JORDAN, Gretchen. Using Logic Models. In: NEWCOMER, Kathryn; HATRY, Harry; WHOLEY, Joseph. Handbook of practical program evaluation. San Francisco: Jossey-Bass, 2015. p. 62-87.

OFICINA DE ATENCIÓN AL DEPORTISTA. Información General. Madrid, 2017. Disponible en: $<$ http://www.oad.es/> Acceso en: 12 may 2017.

PALLARÉS, Susana; AZÓCAR, Fernando; TORREGROSA, Miquel; SELVA, Clara; RAMIS, Yago. Modelos de trayectoria deportiva en waterpolo y su implicación en la transición hacia una carrera profesional alternativa. Cultura Ciencia y Deporte, v. 6, n. 17, p. 93-103, 2011.

PROGRAMA DE ATENCIÓN AL DEPORTISTA DE ALTO NIVEL. Presentación. Madrid, 2017. Disponible en: <https://proad.csd.gob.es/index.php> Acceso en: 12 may 2017.

QUIVY, Rayrnond; CAMPENHOUDT, Luc. Manual de investigación en Ciencias Sociales. Mexico: Limusa, 2005.

REGÜELA, Susana. El projecte personal com a eina per afrontar les transicions de la carrera esportiva. Barcelona: Universitat Autònoma de Barcelona, 2011.

SPARKES, Andrew; SMITH, Brett. Qualitative Research Methods in Sport, Exercise and Health. Oxford: Routledge, 2014.

STAMBULOVA, Natalia; ENGSTRÖM, Cecilia; FRANCK, Alina; LINNÉR, Lukas; LINDAHL, Kent. Searching for an optimal balance: Dual career experiences of Swedish adolescent athletes.

Psychology of Sport and Exercise, v. 21, p. 4-14, 2015. 
STAMBULOVA, Natalia; WYLLEMAN, Paul. Athletes' career development and transitions. In: PAPAIOANNOU, Athanasios; HACKFORT, Dieter. Routledge companion to sport and exercise psychology. London: Routledge, 2014. p. 605-621.

TÁBOAS-PAIS, María Inés; CANALES-LACRUZ, Inma; REY-CAO, Ana; PERICH-SANCHO, María Jesús. El deporte como mecanismo propagandístico de identidad nacional. Movimento, $v$. 21, n. 4, p. $985-997,2015$.

TORREGROSA, Miquel; GONZÁLEZ, María Dolores. Athletes' careers in Spain:

Professionalization and developmental consequences. In: STAMBULOVA, Natalia; RYBA, Tatiana. Athletes' careers across cultures. New York: Routledge, 2013. p. 185-196.

VIANNA, José Antonio; LOVISOLO, Hugo Rodolfo. Social inclusion through sports Project: notes about the evaluation. Movimento, v. 15, n. 3, p.333-354, 2009.

VILANOVA, Anna; PUIG, Nuria. Compaginar la carrera deportiva con la carrera académica para la futura inserción laboral: ¿Una cuestión de estrategia? Revista de Psicología del Deporte, v. 22, n. 1, p. 61-68, 2013.

VILANOVA, Anna; PUIG, Nuria. Personal strategies for managing a second career: The experiences of Spanish Olympians. International Review for the Sociology of Sport, v. 51, n. 5, p. 529-546, 2014.

VON BERTALANFFY, Ludwig. General System Theory. New York: George Braziller, 1968.

WYLLEMAN, Paul; ALFERMANN, Dorothee; LAVALLEE, David. Career transitions in sport: European perspectives. Psychology of Sport and Exercise, v. 5, n. 1, p. 7-20, 2004.

\section{Apoyo:}

Este trabajo forma parte del proyecto "Dual Careers a Catalunya. Buscant sinèrgies entre el sistema educatiu i el sistema Esportiu" (2016 PINEFC 00001), financiado por el Institut Nacional d’Educació Física de Catalunya (INEFC) 